\title{
BMJ Open Biopsychosocial correlates of hope in Asian patients with cancer: a systematic review
}

\author{
Rathi Mahendran, ${ }^{1,2,3}$ Shi Min Chua, ${ }^{1}$ Haikel A Lim, ${ }^{1,3}$ Isaac J Yee, ${ }^{1,4}$ \\ Joyce Y S Tan, ${ }^{1}$ Ee Heok Kua, ${ }^{1,2}$ Konstadina Griva ${ }^{4}$
}

To cite: Mahendran $\mathrm{R}$, Chua SM, Lim HA, et al. Biopsychosocial correlates of hope in Asian patients with cancer: a systematic review. BMJ Open 2016;6:e012087. doi:10.1136/bmjopen-2016012087

- Prepublication history and additional material is available. To view please visit the journal (http://dx.doi.org/ 10.1136/bmjopen-2016012087).

Received 30 March 2016 Revised 21 June 2016 Accepted 31 August 2016

CrossMark

For numbered affiliations see end of article.

\section{Correspondence to} Dr Rathi Mahendran; rathi_mahendran@nuhs. edu.sg

\section{ABSTRACT}

Objective: To examine the factors associated with hope and hopelessness in patients with cancer in Asian countries, and the instruments used to measure hope and hopelessness.

Methords: A comprehensive systematic review was conducted with search terms, including cancer, hope, hopelessness and individual Asian country names, on CINAHL, Embase, PsycINFO, PubMed and Scopus databases. Only quantitative studies on adult cancer populations in Asia examining hope or hopelessness were included.

Results: A total of 2062 unique articles were retrieved from the databases, and 32 studies were selected for inclusion in this review. Hope and hopelessness were most frequently measured with the Herth Hope Index and the Mental Adjustment to Cancer Scale, respectively. The biopsychosocial factors that were most consistently associated with hope and hopelessness included sociodemographic variables (education, employment and economic status); clinical factors (cancer stage, physical condition and symptoms); and psychosocial factors (emotional distress, social support and connections, quality of life, control or self-efficacy, as well as adjustment and resilience).

Discussion: There is a need for more studies from South and Southeast Asia as most studies hailed from East Asia. This review highlighted the possibility of cultural differences influencing factors related to hope, suggesting that cross-cultural studies specifically would facilitate understanding behind these variations, although future reviews on hope should also include studies on hopelessness for a comprehensive understanding of the concept. Finally, more longitudinal research could be conducted to assess whether the factors associated with hope and hopelessness change over time and disease progression.

\section{INTRODUCTION}

Psychological and psychiatric literature have defined hope as a yearning for the amelioration of a dreaded outcome, operationalising it as a positive goal-related (future-oriented)

\section{Strengths and limitations of this study}

- This is the first systematic review conducted that focuses on hope in the Asian cancer population.

- The inclusion of hopelessness in the current review presented a more comprehensive understanding of hope and its antithetical concept in the oncology setting.

- This review attempted to include all articles on hope in patients with cancer in Asian countries, including the non-English articles, which provided a more comprehensive view of the target population.

- This review, however, is limited by the availability of articles. Four articles were not available despite repeated contacts with library and authors, and thus not included.

- Although not a weakness specific to our review, the apparent lack of longitudinal studies conducted in Asian oncology settings may limit the conclusiveness of the directionality of the correlates reported here.

motivational state and a dispositional trait that signalled a tendency to adopt a positive outlook. ${ }^{12}$ Hope, in other words, is a confident, yet uncertain, expectation of achieving a future good that, to the hoping person, is realistically possible, and personally significant. $^{3}$

Hope enables individuals to deal with serious and prolonged threats to their physical and psychological well-being, ${ }^{4}$ and has been established as an important therapeutic factor in medicine and recovery. ${ }^{2} 5$ In oncology settings, it facilitates coping with the cancer diagnosis, ${ }^{6}$ through making and sustaining meaning, ${ }^{1}$ while strengthening resilience regardless of prognosis. ${ }^{7}$ Although hope has not been shown to improve prognosis, ${ }^{8}$ patients without hope (ie, patients who are hopeless) are often depressed and lack the will to live. ${ }^{5}$

Dispositional theories of hope have proposed two components of hopeful thinking: 
pathway thinking, or the ability to conceptualise the means (pathways) through which goals can be achieved; and agency thinking, or the perceived capacity to use such pathways. ${ }^{9}$ Although hope has often been linked to other cognitive and motivational theories, it remains distinct from these constructs. It differs from optimism in its view of goal-directed positive cognitive processes, and self-efficacy in its cross-situational perspective and equal emphasis on agency and pathway thinking. ${ }^{10}$ Agency and pathway components have been associated with better outcomes such as lower levels of depression and anxiety, ${ }^{11}$ better quality of life and physical health, and higher positive affect. ${ }^{12}$

On the other hand, hopelessness has been viewed as an antithetical concept to hope, with both constructs hypothesised to be lying on a continuum rather than being distinctly different. ${ }^{1}{ }^{4}$ Hopelessness is operationalised as a system of negative expectations concerning oneself and one's future life, ${ }^{13}$ or a tendency to lack hopeful thinking. ${ }^{14}$ It is conceptually distinct from concepts such as catastrophising, which refers to tendency to have a negative cognition of focusing on and exaggerating a negative outcome. ${ }^{15}$

To date, there are three reviews on hope in patients with cancer. ${ }^{4} 1216$ The first review, ${ }^{16}$ on articles published between 1982 and 2005, summarised the importance of hope to nurses: levels of hope were not associated with sociodemographic predictors, cancer type and stage, but positively associated with control, coping and spiritual well-being, and negatively associated with physical well-being and fatigue. The second review, ${ }^{12}$ of oncology nursing literature between 2005 and 2009, corroborated these findings: hope was linked to better health and quality of health, higher levels of control, more positive affect, and reduced depressive and anxious symptomatology. The final review updated the literature on hope in oncology up to $2011,{ }^{4}$ and included perspectives of caregivers, family members and healthcare professionals. The review confirmed earlier findings that hope reduced the impact of psychological distress and fatigue in patients.

As the earlier reviews were conducted without clear adherence to either of the gold-standard Quality of Reporting of Meta-Analyses (QUOROM) ${ }^{17}$ or Preferred Reporting Items for Systematic Reviews and Meta-Analyses (PRISMA) ${ }^{18}$ guidelines, they do not lend themselves well to replication. Also, the reviews by $\mathrm{Chi}^{16}$ and $\mathrm{Butt}^{12}$ may not have been exhaustive because they focused primarily on hope, and not its antithetical concept of hopelessness. In addition, the review by Olver ${ }^{4}$ included studies on hope in patients as reported by proxies, namely caregivers and healthcare professionals, which may not be a true reflection of the perspectives of hope in patients with cancer. Furthermore, only articles published in English were examined, with the authors themselves acknowledging the inadequate investigation into the cross-cultural validity of these findings.
Comparative studies in the UK have suggested cultural variation in the concept of hope, noting higher levels of hopelessness in patients with cancer of Asian background than their Caucasian counterparts. ${ }^{19} 20$ Aside from differences in understanding and conceptualising hope, ${ }^{21}{ }^{22}$ patients of Eastern origin more commonly attribute illness to predestined causes than patients of Western origin. ${ }^{23}$ As these cultural differences could be due to differences in socioeconomic and education level ${ }^{20}$ or the migration effects and position of immigrants as outsiders within a national health service, ${ }^{23}$ there exists a need to examine hope and hopelessness in Asian patients with cancer within Asian countries to better understand the concept in this population.

\section{The present study}

The growth of Asian populations, increased prevalence of cancer ${ }^{24}$ and significant psychiatric sequelae in this population $^{25}$ call for a more nuanced appreciation of hope in culturally unique Asian settings ${ }^{26} 27$ to guide the development of culturally relevant support services for oncology patients in Asia. Thus, the objectives of the present systematic review are to examine (1) the instruments used to measure hope and hopelessness and (2) the biopsychosocial factors associated with hope and hopelessness in patients with cancer in Asian countries.

\section{METHODS}

This review adhered to the PRISMA guidelines (see online supplementary file 1). ${ }^{18}$ The review protocol can be retrieved from the PROSPERO International Prospective Register of Systematic Review. ${ }^{28}$

\section{Search strategy}

For the purposes of this review, (1) hope and hopelessness were conceptualised as lying on a continuum or antithetical, but not separate, and (2) Asia was defined as countries in the East, South and Southeast Asia for cultural homogeneity.

Articles were retrieved from CINAHL, Embase, PsycINFO, PubMed and Scopus databases from inception to May 2015. Reference lists of relevant articles were searched by hand to include additional articles not captured by the database searches. The following search terms were applied: cancer; oncology; tumor; neoplasm; carcinoma; malignant; sarcoma; Asia; Burma; Cambodia; Vietnam; Japan; Korea; Mongolia; Thailand; Singapore; China; India; Malaysia; Indonesia; Laos; Myanmar; Philippines; Bangladesh; Taiwan; Hong Kong; Pakistan; Sri Lanka; hope; hopelessness (see online supplementary file 2). No language or date restrictions were imposed, although all foreign language articles had English titles. The 3174 items from all searches (including reference lists) were exported into EndNote X7; 2062 unique entries remained after the removal of duplicates. 


\section{Study selection criteria}

Two authors independently and conservatively subjected the titles and abstracts of the 2062 entries to the following inclusion criteria (agreement $\alpha=0.90$ ): (1) peerreviewed journal articles, to ensure the quality of research; (2) only primary quantitative research investigating the correlates of hope and hopelessness (as earlier defined); (3) oncology populations in Asian countries (as previously defined); and (4) adult populations (defined as 18 years old and above). Owing to the exploratory nature of this review, no cancer site or stage restrictions were imposed. Studies were excluded if they (1) were qualitative or scale validation studies; (2) recruited children, adolescents or cancer survivors; or (3) used family members, caregivers or healthcare professionals as proxies of patients' perspectives.

\section{Data selection and extraction}

Of the 2062 entries, 65 were selected for a full-text review. Four potentially relevant entries ${ }^{29-32}$ were not included because the full-text articles (in non-English language journals) were unobtainable despite repeated library requests and attempts at contacting authors. The remaining 61 full-text articles, including non-English articles, were reviewed for eligibility based on the inclusion criteria independently by two authors with a fluent command of the publication language. A consensus between three authors was necessary when there was disagreement between the two authors (agreement $\alpha=0.90$ ).

A total of 32 journal articles were included in the review. The quality of each study was assessed by two reviewers with the modified STROBE checklist, ${ }^{33}$ which consists of 18 items examining study design, participants, statistical analysis, results, limitations, outcomes and study generalisability. Items were scored 0 (not done), 1 (done partially) and 2 (done well), with double scores for statistical methods and outcomes. Total scores range from 0 to 40 . Study quality was then rated as low, moderate or high according to the tertile of scores. The quality of all identified studies was found to be either moderate or high, and they were included in the current review.

Data extracted from included articles comprised (1) study design; (2) patient characteristics; (3) measurement of hope or hopelessness; and (4) factors related to hope or hopelessness (including the measurements used and the relationship between the factors and hope or hopelessness). The flow diagram of the study selection is presented in figure 1 .

To summarise the state of the literature for each identified variable, a summary code was applied to each factor, as suggested by Sallis $e t$ al. ${ }^{34}$ The percentage of findings supporting each association with hope or
Figure 1 PRISMA flow diagram of study selection. PRISMA, Preferred Reporting Items for Systematic Reviews and Meta-Analyses.

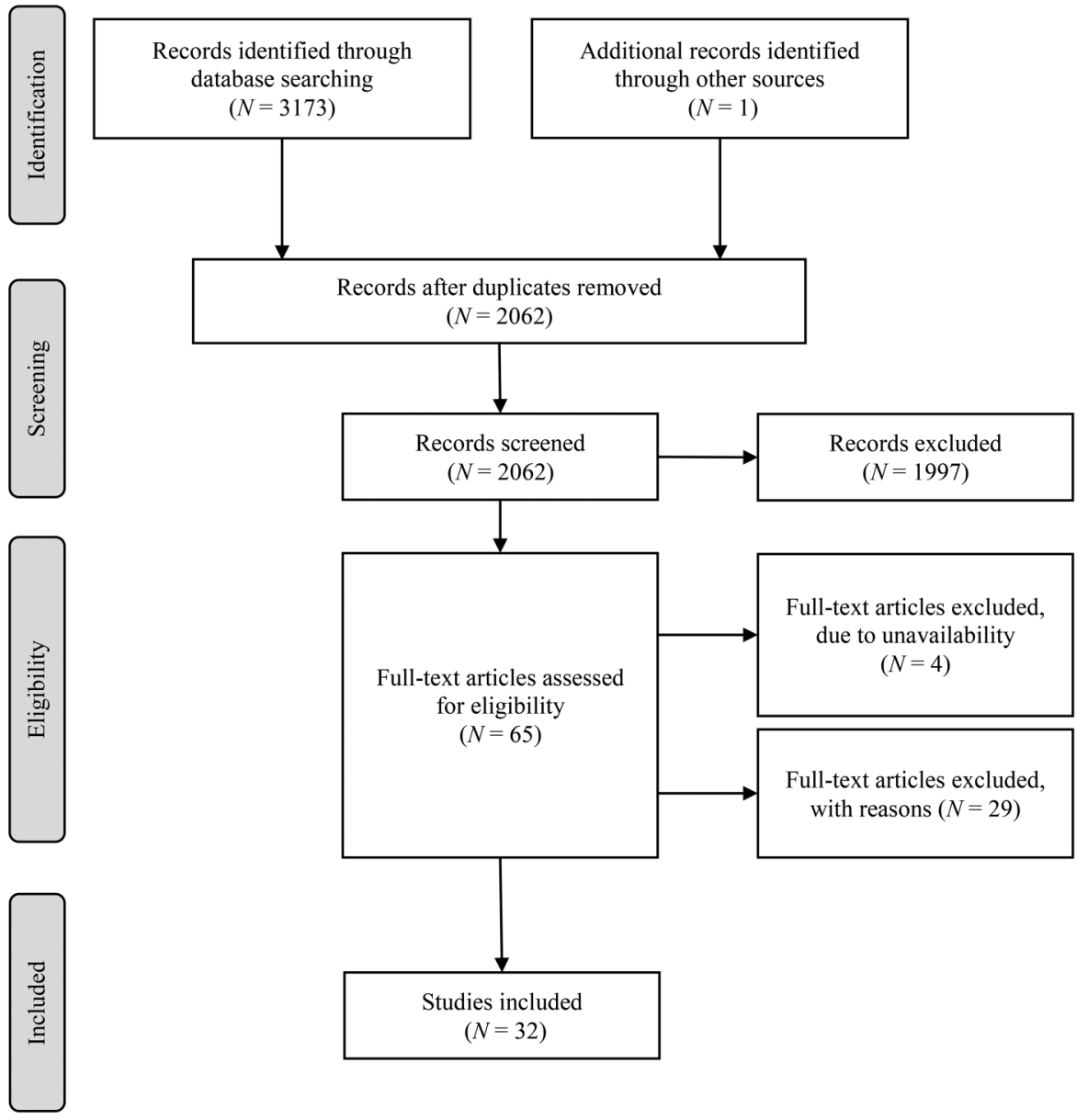


hopelessness was calculated by the number of studies supporting the expected association divided by the total number of studies examining the factor. Based on this percentage, the variable will be classified as: no association, indeterminate or inconsistent, or positive or negative association (see table 1 ).

\section{RESULTS}

\section{Characteristics of included studies}

The majority of the identified studies $(\mathrm{N}=28)$ were crosssectional, while four were longitudinal studies. ${ }^{35-38}$ Sample sizes varied from $50^{39}$ to 1334 participants. ${ }^{40}$ A total of 11 studies were based on mixed samples of patients with various cancer types, nine were on breast cancer $^{41-49}$ and six were on lung cancer. ${ }^{36-38} 405051$ The remaining studies involved participants diagnosed with haemolymph neoplasm, ${ }^{52}$ nasopharyngeal carcinoma, ${ }^{15}$ colorectal cancer, ${ }^{35}$ oral cavity cancer, ${ }^{39}$ oesophageal cancer $^{53}$ and cervical cancer. ${ }^{54}$ Almost all studies $(\mathrm{N}=31)$ involved various cancer stages; only one study exclusively involved patients with recurrent or metastatic cancer. ${ }^{45}$

The majority of the studies $(\mathrm{N}=31)$ were conducted in East Asia, with 3 from mainland China, ${ }^{46} 53542$ from Hong Kong, ${ }^{35} 3911$ from Taiwan, ${ }^{15} 415152{ }^{55-61} 8$ from Korea $^{42-44} 484962-64$ and 7 from Japan. ${ }^{36-38} 40 \quad 45 \quad 5065$ Only one study was conducted in Southeast Asia (Malaysia). ${ }^{47}$

Table 2 presents a summary of the studies included in this review.

\section{Measurements of hope and hopelessness}

Hope and hopelessness were examined in 20 and 12 studies, respectively. State hope was measured in 18 studies, with the majority $(\mathrm{N}=12)$ using the Herth Hope Index (HHI), ${ }^{3}$ four using the Nowotny Hope Scale $(\mathrm{NHS})^{66}$ and two using the Hope Scale by Kim and Lee (KLHS) ${ }^{67}$ Trait hope was measured in two studies with the Snyder Hope Scale (SHS) ${ }^{68}$ State hopelessness was measured in 11 studies; seven used the Mental Adjustment to Cancer Scale (MAC), ${ }^{69}$ one used the short version of the MAC (Mini-MAC) ${ }^{70}$ and three used the Beck Hopelessness Scale (BHS) ${ }^{13}$ Trait hopelessness was measured in one study with the Short Interpersonal Reactions Inventory (SIRI).$^{71}$

\begin{tabular}{lll}
$\begin{array}{l}\text { Table } 1 \\
\text { association of variables }\end{array}$ & \multicolumn{2}{l}{ Summary codes for strength of evidence of } \\
$\begin{array}{l}\text { \% of studies } \\
\text { supporting } \\
\text { association }\end{array}$ & $\begin{array}{l}\text { Summary } \\
\text { code }\end{array}$ & Meaning of code \\
\hline $0-33$ & 0 & $\begin{array}{l}\text { No association } \\
\text { Indeterminate, } \\
\text { inconsistent }\end{array}$ \\
$60-59$ & $?$ & $\begin{array}{l}\text { Positive association } \\
\text { Negative association }\end{array}$ \\
\hline
\end{tabular}

Factors associated with hope and hopelessness

Table 3 provides a summary of the factors associated with hope and hopelessness.

\section{Sociodemographic variables}

Sociodemographic correlates of hope and hopelessness examined include age, gender, marital status, education, employment and economic status, religion and race.

Education, employment and economic status were more consistently associated with hope and hopelessness. Twelve studies examined education, with significant associations reported in 10 studies; education was positively correlated with hope ${ }^{41} 4252586062$ and negatively associated with hopelessness. ${ }^{37} 384753$ Six of 10 studies reported significant associations between employment status and hope; patients who were employed had higher hope ${ }^{42} 52 \quad 5860$ and lower hopelessness. ${ }^{45} 57$ Economic status was a significant correlate in three of five studies; hope was correlated with higher income, ${ }^{46}{ }^{49}$ while patients who required financial support reported lower hope. ${ }^{52}$

The associations with age, gender, religion and race were less consistent. Significant associations were reported in only 6 of 14 studies; age was negatively associated with hope ${ }^{52} 5562$ and positively correlated with hopelessness. ${ }^{36} 3753$ Of the 10 studies that examined religion, 4 studies reported significant associations; religious participation was associated with higher hope $^{41} 4955$ and lower hopelessness. ${ }^{47}$ With regards to religious affiliations, hope was associated with Christianity ${ }^{41} 55$ and Buddhism. ${ }^{55}$ Race was identified in one study, but the direction of this association was not reported. ${ }^{47}$ Of the three studies examining gender, only one study reported gender to be a significant factor; women had significantly higher levels of hope than men in a long-term care hospital while no significant results were found for patients in a general hospital. ${ }^{62}$

A majority of the 12 studies examining marital status reported no significant associations, with the exception of three studies; married patients had higher hope than patients who were unmarried ${ }^{39} 55$ or separated. ${ }^{41}$

\section{Clinical factors and outcomes}

The clinical correlates associated with hope and hopelessness include cancer stage and type, awareness of diagnosis, treatment-related factors, physical condition, symptoms and clinical markers or end points.

Cancer stage, physical condition and symptoms were consistently associated with hope and hopelessness. Eight of 12 studies examining cancer stage reported significant associations; hope was associated with early stage ${ }^{4162}$ and localised, non-metastatic cancer, ${ }^{55}$ while state and trait hopelessness were associated with advanced cancer stages. ${ }^{36-38} 4753$ Physical condition was a significant factor reported in 8 of 11 studies; these indicated hope with better physical health and functioning, ${ }^{35}{ }^{49}$ better performance status ${ }^{15}$ and lower interference with functioning, ${ }^{55}$ and hopelessness with poorer performance 


\begin{tabular}{|c|c|c|c|c|c|c|c|c|c|}
\hline \multirow[b]{2}{*}{ No. } & \multirow[b]{2}{*}{ Study } & \multirow[b]{2}{*}{ Country } & \multirow[b]{2}{*}{ Type of cancer } & \multirow[b]{2}{*}{$\begin{array}{l}\text { Cancer } \\
\text { stage }\end{array}$} & \multirow[b]{2}{*}{$\begin{array}{l}\text { Patients } \\
\text { (\% male) }\end{array}$} & \multicolumn{2}{|l|}{ Age } & \multirow[b]{2}{*}{$\begin{array}{l}\text { Instruments } \\
\text { (hope/ } \\
\text { hopelessness) }\end{array}$} & \multirow[b]{2}{*}{ Instruments (other variables) } \\
\hline & & & & & & $\begin{array}{l}\text { Mean age } \\
\text { (SD)/age } \\
\text { group (\%) }\end{array}$ & $\begin{array}{l}\text { Age } \\
\text { range }\end{array}$ & & \\
\hline 1 & $\begin{array}{l}\text { Hwang } \\
\text { et } a f^{41}\end{array}$ & Taiwan & Breast cancer & Various & $120(0 \%)$ & $\begin{array}{l}41.79 \\
(9.83)\end{array}$ & $20-66$ & NHS (Mandarin) & $\begin{array}{l}\text { Mishel's Uncertainty in Illness Scale, and the } \\
\text { Cohen's Interpersonal Support Evaluation List } \\
\text { (Mandarin) }\end{array}$ \\
\hline 2 & $\begin{array}{l}\text { Chen and } \\
\text { Wang } \\
52\end{array}$ & Taiwan & $\begin{array}{l}\text { Haemolymph } \\
\text { neoplasm }\end{array}$ & Various & $\begin{array}{l}75 \\
(54.7 \%)\end{array}$ & $\begin{array}{l}40-49 \\
(30.7 \%)\end{array}$ & $20-60$ & HHI (Mandarin) & $\begin{array}{l}\text { Personal Resource Questionnaire } 85 \text { Part-II } \\
\text { (Mandarin) }\end{array}$ \\
\hline 3 & $\operatorname{Lee}^{44}$ & Korea & Breast cancer & Various & $122(0 \%)$ & $\begin{array}{l}44.40 \\
(7.62)\end{array}$ & $27-63$ & $\mathrm{HHI}$ & $\begin{array}{l}\text { Psychological adjustment to Breast Cancer } \\
\text { Factor, Piper Fatigue Scale }\end{array}$ \\
\hline 4 & $\begin{array}{l}\text { Chang and } \\
\mathrm{Li}^{55}\end{array}$ & Taiwan & Various & Various & 137 (NA) & $\begin{array}{l}51.9 \\
(15.76)\end{array}$ & 19-84 & NHS (Mandarin) & $\begin{array}{l}\text { Symptom distress scale, Physical } \\
\text { self-maintenance scale and the Perception of } \\
\text { control scale (Mandarin versions) }\end{array}$ \\
\hline 5 & Chen $^{56}$ & Taiwan & Various & Various & $\begin{array}{l}226 \\
(48.7 \%)\end{array}$ & NR & $\geq 18$ & HHI (Mandarin) & $\begin{array}{l}\text { Pain Assessment Form, Perceived Meaning of } \\
\text { Cancer Pain Inventory and Karnofsky } \\
\text { Performance Scale (Mandarin) }\end{array}$ \\
\hline 6 & Hsu et $a^{\rho^{1}}$ & Taiwan & Lung cancer & Various & 164 (NA) & NR & $\geq 18$ & $\mathrm{HHI}$ (Mandarin) & $\begin{array}{l}\text { Brief Pain Inventory, and the Mishel Uncertainty } \\
\text { Illness Scale (Mandarin) }\end{array}$ \\
\hline 7 & Lai et $a l^{15}$ & Taiwan & $\begin{array}{l}\text { Nasopharyngeal } \\
\text { carcinoma }\end{array}$ & Various & $\begin{array}{l}115 \\
(76.5 \%)\end{array}$ & $\begin{array}{l}40-59 \\
(59.1 \%)\end{array}$ & $\geq 20$ & $\mathrm{HHI}$ (Mandarin) & $\begin{array}{l}\text { Symptom Distress Scale-modified, and Coping } \\
\text { Strategies Questionnaire-Catastrophising-Dis } \\
\text { (Mandarin) }\end{array}$ \\
\hline 8 & Lin et $a^{{ }^{99}}$ & Taiwan & Various & Various & $\begin{array}{l}484 \\
(47.7 \%)\end{array}$ & $\begin{array}{l}\text { With pain: } \\
58.06 \\
(14.52) \\
\text { Without } \\
\text { pain: } 58.50 \\
(14.77)\end{array}$ & $\geq 18$ & HHI (Mandarin) & $\begin{array}{l}\text { Brief pain Inventory, and the Karnorfsy } \\
\text { Performance Scale (Mandarin) }\end{array}$ \\
\hline 9 & Lin et $a^{58}$ & Taiwan & Various & Various & $\begin{array}{l}124 \\
(47.6 \%)\end{array}$ & $\begin{array}{l}57.50 \\
(13.10)\end{array}$ & 24-89 & $\mathrm{HHI}$ (Mandarin) & $\begin{array}{l}\text { Multidimensional Health Locus of Control } \\
\text { Scales, Demographics and Disease sheet }\end{array}$ \\
\hline 10 & $\begin{array}{l}\text { Uchitomi } \\
\text { et } a \beta^{\beta 8}\end{array}$ & Japan & Lung cancer & Various & $\begin{array}{l}205 \\
(60.0 \%)\end{array}$ & $61.9(10.9)$ & $22-83$ & MAC (Japanese) & $\begin{array}{l}\text { Structured Clinical Interview for DSM-III-R, } \\
\text { Eysenck Personality Questionnaire-Revised and } \\
\text { a 4-point verbal scale for pain and dyspnoea } \\
\text { (Japanese) }\end{array}$ \\
\hline 11 & $\begin{array}{l}\text { Jo and } \\
\text { Son }^{42}\end{array}$ & Korea & Breast cancer & Various & $113(0 \%)$ & $\begin{array}{l}40-50 \\
(41.6 \%)\end{array}$ & $21-70$ & NHS (Korean) & $\begin{array}{l}\text { Mishel Uncertainty in Illness Scale, Ro's Korean } \\
\text { Quality of Life Scale (Korean) }\end{array}$ \\
\hline 12 & $\begin{array}{l}\text { Lin and } \\
\text { Tsay }^{60}\end{array}$ & Taiwan & Various & Various & $\begin{array}{l}124 \\
(47.6 \%)\end{array}$ & $\begin{array}{l}57.50 \\
(13.10)\end{array}$ & 24-89 & $\mathrm{HHI}$ & Multidimensional Health Locus of Control \\
\hline 13 & $\begin{array}{l}\text { Nagano } \\
\text { et } a^{\beta 6}\end{array}$ & Japan & Lung cancer & Various & $68(74 \%)$ & $>60(38 \%)$ & $\leq 70$ & SIRI (Japanese) & - \\
\hline 14 & $\begin{array}{l}\text { Nakaya } \\
\text { et } a^{\beta 7}\end{array}$ & Japan & Lung cancer & Various & $\begin{array}{l}1178 \\
(71 \%)\end{array}$ & $64(9)$ & NA & MAC (Japanese) & $\begin{array}{l}\text { Eysenck Personality Questionnaire-Revised, } \\
\text { Hospital Anxiety and Depression Scale } \\
\text { (Japanese) }\end{array}$ \\
\hline
\end{tabular}




\begin{tabular}{|c|c|c|c|c|c|c|c|c|c|}
\hline 20 & $\begin{array}{l}\text { Shim and } \\
\mathrm{Hahm}^{64}\end{array}$ & Korea & Various & Various & $\begin{array}{l}131 \\
(60.3 \%)\end{array}$ & $52.5(12.1)$ & NA & $\begin{array}{l}\text { Mini-MAC } \\
\text { (Korean) }\end{array}$ & $\begin{array}{l}\text { Hospital Anxiety and Depression Scale, } \\
\text { Schedule of Attitudes towards Hastened Death, } \\
\text { Assessment of Chronic Illness Therapy-Spiritual } \\
\text { Well-being scale and European Organization for } \\
\text { Research and Treatment of Cancer Quality of } \\
\text { Life Questionnaire (Korean) }\end{array}$ \\
\hline 21 & Shun et $a \rho^{\beta 1}$ & Taiwan & Various & Various & $\begin{array}{l}182 \\
(46 \%)\end{array}$ & $\begin{array}{l}50.81 \\
(10.4)\end{array}$ & $21-78$ & HHI (Mandarin) & $\begin{array}{l}\text { Fatigue Symptom Inventory, and the Karnofsky } \\
\text { Performance Status (Mandarin) }\end{array}$ \\
\hline 22 & $\begin{array}{l}\text { Ueta and } \\
\text { Onishi }\end{array}$ & Japan & Breast cancer & $\begin{array}{l}\text { Recurrent } \\
\text { cancer/ } \\
\text { metastasis }\end{array}$ & $64(0 \%)$ & $\begin{array}{l}58.33 \\
(11.28)\end{array}$ & $33-82$ & MAC (Japanese) & $\begin{array}{l}\text { Visual analogue scale, Tri-axial Coping Scale } \\
\text { (TAC-24) (Japanese) }\end{array}$ \\
\hline 23 & Lee et $a^{57}$ & Taiwan & Various & Various & $\begin{array}{l}234 \\
(35.5 \%)\end{array}$ & $\begin{array}{l}51-65 \\
(44.9 \%)\end{array}$ & NA & BHS (Mandarin) & $\begin{array}{l}\text { Demoralisation Scale-Mandarin Version, Patient } \\
\text { Health Questionnaire, McGill Quality of Life } \\
\text { Questionnaire-Taiwanese version }\end{array}$ \\
\hline 24 & $\begin{array}{l}\text { Jun and } \\
\mathrm{Ko}^{63}\end{array}$ & Korea & Various & Various & $\begin{array}{l}120 \\
(40.0 \%)\end{array}$ & $\begin{array}{l}55.17(\mathrm{NA}) \\
45-65 \\
(60.0 \%)\end{array}$ & $\geq 22$ & $\begin{array}{l}\text { Hope Scale by } \\
\text { Kim and Lee }\end{array}$ & $\begin{array}{l}\text { Fatigue Scale by Mendoza et al, and the } \\
\text { Spiritual Well-being Scale by Paloutzian and } \\
\text { Ellisonn }\end{array}$ \\
\hline 25 & $\begin{array}{l}\text { Shimizu } \\
\text { et } a{ }^{40}\end{array}$ & Japan & Lung cancer & Various & $\begin{array}{l}1334 \\
(71.4 \%)\end{array}$ & 64.2 (NA) & $26-88$ & MAC (Japanese) & $\begin{array}{l}\text { Hospital Anxiety and Depression Scale, and the } \\
\text { Eysenck Personality Questionnaire-Revised } \\
\text { (Japanese) }\end{array}$ \\
\hline
\end{tabular}




\begin{tabular}{|c|c|c|c|c|c|c|c|c|c|}
\hline \multirow[b]{2}{*}{ No. } & \multirow[b]{2}{*}{ Study } & \multirow[b]{2}{*}{ Country } & \multirow[b]{2}{*}{ Type of cancer } & \multirow[b]{2}{*}{$\begin{array}{l}\text { Cancer } \\
\text { stage }\end{array}$} & \multirow[b]{2}{*}{$\begin{array}{l}\text { Patients } \\
\text { (\% male) }\end{array}$} & \multicolumn{2}{|l|}{ Age } & \multirow[b]{2}{*}{$\begin{array}{l}\text { Instruments } \\
\text { (hope/ } \\
\text { hopelessness) }\end{array}$} & \multirow[b]{2}{*}{ Instruments (other variables) } \\
\hline & & & & & & $\begin{array}{l}\text { Mean age } \\
\text { (SD)/age } \\
\text { group (\%) }\end{array}$ & $\begin{array}{l}\text { Age } \\
\text { range }\end{array}$ & & \\
\hline 26 & Tae et $a{ }^{49}$ & Korea & Breast cancer & Various & $214(0 \%)$ & $\begin{array}{l}41-50 \\
(38 \%)\end{array}$ & $\geq 18$ & HHI (Korean) & $\begin{array}{l}\text { Zung Self-rating Depression Scale, Rosenberg } \\
\text { Self-Esteem Scale, Health Self-rating Scale in } \\
\text { Health and Activity Survey, Kang's Family } \\
\text { Support Scale and visual analogue scales } \\
\text { measuring pain and fatigue (Korean) }\end{array}$ \\
\hline 27 & $\begin{array}{l}\text { Chae and } \\
\text { Kim }^{62}\end{array}$ & Korea & Various & Various & $\begin{array}{l}175 \\
(55.3 \%)\end{array}$ & $\begin{array}{l}<60 \\
(56.0 \%)\end{array}$ & NA & $\begin{array}{l}\text { Hope Scale by } \\
\text { Kim and Lee }\end{array}$ & Cobb Family Support Scale \\
\hline 28 & Han et $a^{53}$ & China & $\begin{array}{l}\text { Oesophageal } \\
\text { cancer }\end{array}$ & Various & $\begin{array}{l}301 \\
(72.0 \%)\end{array}$ & $\begin{array}{l}\text { Male: } \\
60.71 \\
(10.9) \\
\text { Female: } \\
57.64 \\
(10.9)\end{array}$ & NA & BHS (Mandarin) & $\begin{array}{l}\text { Center for Epidemiological Studies Depression } \\
\text { Scale, Multidimensional Scale of Perceived } \\
\text { Social Support (Mandarin) }\end{array}$ \\
\hline 29 & $\begin{array}{l}\text { Horii and } \\
\text { Maekawa }^{50}\end{array}$ & Japan & Lung cancer & Various & $\begin{array}{l}203 \\
(69.95 \%)\end{array}$ & $65.6(10.0)$ & $\geq 20$ & MAC (Japanese) & $\begin{array}{l}\text { Life Adjustment Scale for Patients with Lung } \\
\text { Cancer, Tangible Assistance Scale, Eastern } \\
\text { Cooperative Oncology Group Performance } \\
\text { Status and the Medical Outcome Study Short } \\
\text { Form-9 (Japanese) }\end{array}$ \\
\hline 30 & $\begin{array}{l}\text { Ryu and } \\
\mathrm{Yi}^{48}\end{array}$ & Korea & Breast cancer & Various & $163(0 \%)$ & 51.5 (NA) & $36-67$ & NHS & $\begin{array}{l}\text { Resilience Scale by Wagnild, Spousal Support } \\
\text { Scale by Nam and the Quality of Life Scale by } \\
\text { Ferrell }\end{array}$ \\
\hline 31 & $\begin{array}{l}\text { Raja } \\
\text { Lexshimi } \\
\text { et } a l^{47}\end{array}$ & Malaysia & Breast cancer & Various & $216(0 \%)$ & $\begin{array}{l}54.57 \\
(11.00)\end{array}$ & NA & $\begin{array}{l}\text { MAC (Malay and } \\
\text { English) }\end{array}$ & $\begin{array}{l}\text { Functional Assessment of Chronic Illness } \\
\text { Therapy-Spiritual Well-Being (Malay or English) }\end{array}$ \\
\hline 32 & Yang et $a^{54}$ & China & Cervical cancer & & $224(0 \%)$ & $\begin{array}{l}49.16 \\
(10.11)\end{array}$ & 22-79 & $\mathrm{HHI}$ (Mandarin) & $\begin{array}{l}\text { Hospital Anxiety and Depression Scale, Life } \\
\text { Orientation Scale-Revised and the General } \\
\text { Self-Efficacy Scale (Mandarin) }\end{array}$ \\
\hline
\end{tabular}

BHS, Beck Hopelessness Scale; HHI, Herth Hope Inventory; KLHS, Kim and Lee Hope Scale; MAC, Mental Adjustment to Cancer Scale, Mini-MAC, Shortened MAC Scale; NHS, Nowotny Hope Scale; NR, not reported; SHS, Snyder's Hope Scale; SIRI, Short Interpersonal Reactions Inventory. 


\begin{tabular}{|c|c|c|c|c|c|c|c|c|c|}
\hline \multirow[b]{2}{*}{ Factors } & \multirow{2}{*}{$\begin{array}{l}\text { \# of studies } \\
\text { examining } \\
\text { associations }\end{array}$} & \multicolumn{3}{|c|}{$\begin{array}{l}\text { Associations with } \\
\text { hope }^{\star}\end{array}$} & \multicolumn{3}{|c|}{$\begin{array}{l}\text { Associations with } \\
\text { hopelessness }\end{array}$} & \multicolumn{2}{|c|}{ Summary code } \\
\hline & & +ve & -ve & $?$ & +ve & $-\mathbf{v e}$ & $?$ & with hopet & \% Studies (N) \\
\hline \multicolumn{10}{|l|}{ Sociodemographic variables } \\
\hline Age (older) & 14 & & 3 & & 3 & & & ?? & $42.9(6 / 14)$ \\
\hline Gender (female) & 3 & 1 & & & & & & $?$ & $33.33(1 / 3)$ \\
\hline Marital status (married) & 12 & 3 & & & & & & 0 & $25.00(3 / 12)$ \\
\hline Education (education level) & 12 & 6 & & & & 4 & & ++ & $83.33(10 / 12)$ \\
\hline Employment (employed) & 10 & 4 & & & & 2 & & ++ & $60.00(6 / 10)$ \\
\hline Economic status & 5 & 3 & & & & & & + & $60.00(3 / 5)$ \\
\hline Religion & 10 & & & & & & & ?? & $40.00(4 / 10)$ \\
\hline Participation & & 3 & & & & 1 & & & \\
\hline Affiliation (Christianity) & & 2 & & & & & & & \\
\hline Affiliation (Buddhism) & & 1 & & & & & & & \\
\hline Race & 1 & & & & & & 1 & $?$ & $100.00(1 / 1)$ \\
\hline \multicolumn{10}{|l|}{ Clinical variables } \\
\hline Cancer stage & 11 & & 3 & & 5 & & & -- & $72.72(8 / 11)$ \\
\hline Cancer type & 6 & & & & 1 & & 1 & $?$ & $33.33(2 / 6)$ \\
\hline Awareness of diagnosis & 3 & 2 & & & 1 & & & ?? & $100.00(3 / 3)$ \\
\hline Treatment & 9 & & & & & & & ?? & $66.67(6 / 9)$ \\
\hline Surgery & & 2 & & & & & & & \\
\hline $\begin{array}{l}\text { Combination of chemotherapy and } \\
\text { radiotherapy }\end{array}$ & & 1 & & & & & & & \\
\hline Frequency and duration & & & 1 & & & & & & \\
\hline Outpatients & & 1 & & & & & & & \\
\hline Type of surgery & & & & & & & 1 & & \\
\hline Physical condition & 11 & 5 & & & & 3 & & ++ & $72.73(8 / 11)$ \\
\hline Symptoms & 11 & & 8 & & 3 & & & -- & $100.00(11 / 11)$ \\
\hline Mortality & 2 & & & & 2 & & & - & $100.00(2 / 2)$ \\
\hline Immunity & 1 & & & & & 1 & & + & $100.00(1 / 1)$ \\
\hline \multicolumn{10}{|l|}{ Psychosocial variables } \\
\hline Emotional distress & 9 & & 5 & & 4 & & & -- & $100.00(9 / 9)$ \\
\hline Demoralisation and resignation & 3 & & & & 3 & & & - & $100.00(3 / 3)$ \\
\hline Quality of life & 5 & 3 & & & & 2 & & ++ & $100.00(5 / 5)$ \\
\hline Adjustment and resilience & 4 & 3 & & & & 1 & & ++ & $100.00(4 / 4)$ \\
\hline Coping responses & 4 & 3 & & & & 1 & & ++ & $100.00(4 / 4)$ \\
\hline Uncertainty & 3 & & 3 & & & & & - & $100.00(3 / 3)$ \\
\hline Control and self-efficacy & 5 & 4 & & & & 1 & & ++ & $100.00(5 / 5)$ \\
\hline Self-esteem & 2 & 2 & & & & & & + & $100.00(2 / 2)$ \\
\hline
\end{tabular}




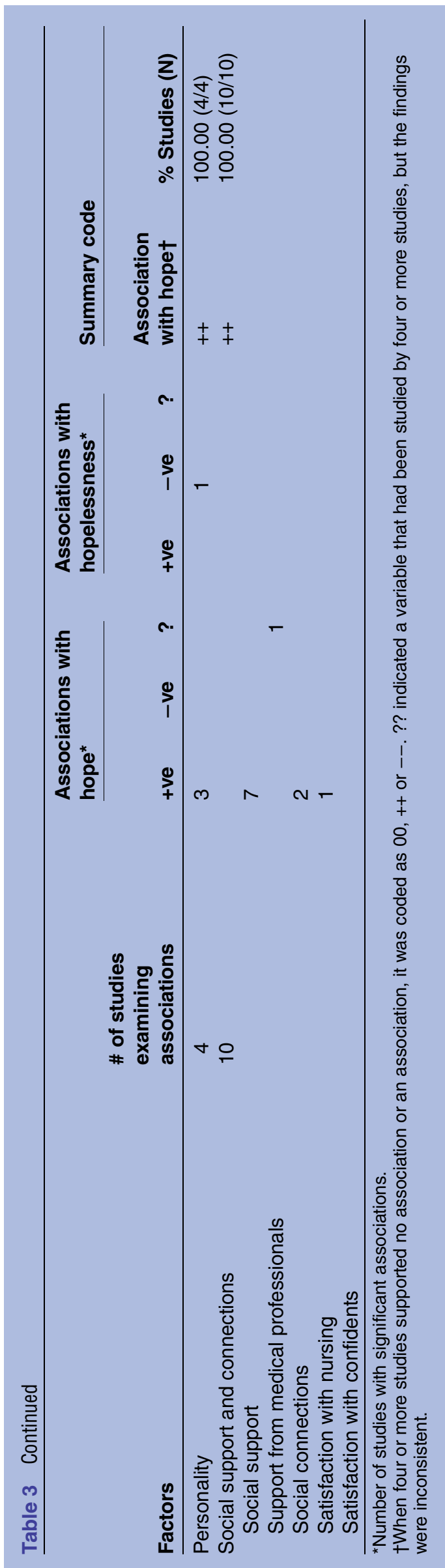

status. $^{37} 3865$ Eleven studies examined the associations between experience of symptoms and hope or hopelessness; pain and fatigue were more frequently measured and showed consistent associations with hope and hopelessness. Hope was associated with bearable pain intensity, ${ }^{56}$ lower pain severity ${ }^{51}$ and lower pain interference in daily life. ${ }^{51} 59$ Hope was also negatively correlated to fatigue, ${ }^{49}{ }^{63}$ fatigue intensity, ${ }^{15}$ duration of fatigue and fatigue-related interference. ${ }^{61}$ On the other hand, hopelessness was associated with pain severity, ${ }^{37} 65$ dyspnoea $^{37}{ }^{38}$ and the presence of other symptoms. ${ }^{45}$

Significant associations were reported in all three studies that examined awareness of diagnosis, ${ }^{53} 5860$ but the results were not consistent. In the studies included, $\sim 58 \%^{53}$ to $79 \%^{58} 60$ of the patients were aware of their cancer diagnoses, while the remaining were either not aware or partially aware of the diagnoses due to physicians' or family members' decisions to conceal information about the diagnosis. While two studies reported that awareness of diagnosis was associated with higher hope ${ }^{5860}$ patients who were aware of their diagnoses felt more hopeless than those unaware in another study. ${ }^{53}$

Treatment-related factors (ie, type of treatment, duration dose and location) were examined in nine studies, and six studies found significant associations between these treatment-related factors and hope or hopelessness. ${ }^{41} 4757586062$ These studies, however, examined different treatment-related factors, making results indeterminate. Patients who only underwent surgery reported higher hope than those who underwent only chemotherapy, or chemotherapy and surgery. ${ }^{41} 62$ Patients who underwent a combination of chemotherapy and radiotherapy also reported higher hope than those who underwent either chemotherapy or radiotherapy alone. ${ }^{62}$ Frequency and duration of chemotherapy treatment was negatively correlated with hope. ${ }^{41}$ In addition, outpatients reported higher hope than inpatients. ${ }^{58} 60$ Hopelessness was significantly correlated with type of surgery in patients with breast cancer. ${ }^{47}$

Associations between clinical markers or end points were less conclusive as they were only assessed in three studies. ${ }^{36} 377^{43}$ State and trait hopelessness were related to increased mortality risk ${ }^{36}$ and weaker cellular immunity. ${ }^{43}$

Only two of six studies reported significant associations with cancer type ${ }^{57}$ and histologic type, ${ }^{37}$ making associations indeterminate. While it was not indicated which cancer type was correlated with hopelessness, ${ }^{57}$ patients with squamous cell carcinoma reported being more hopeless. ${ }^{37}$

\section{Psychosocial correlates}

The psychosocial factors identified could be classified into the following themes: emotional distress, demoralisation and resignation, quality of life, adjustment and resilience, coping responses, uncertainty, control and self-efficacy, self-esteem, personality, as well as social support and connections. 
The current review presented the strongest evidence for emotional distress as a psychological correlate of hope and hopelessness. All nine studies that examined emotional distress reported significant associations. ${ }^{15} 353840434953-55$ State hope was negatively associated with depression, ${ }^{49} 54$ anxiety $^{54}$ and symptom distress. ${ }^{155}$ Trait hope was associated with the trajectory of depression and anxiety; patients who had significant decrease in distress over time were more likely to demonstrate higher trait hope than those who showed maintenance of high distress over time. ${ }^{35}$ Hopelessness was positively correlated to depression ${ }^{40} 43$ and anxiety. ${ }^{53}$ History of depression also significantly predicted hopelessness at 3 months follow-up. ${ }^{38}$ Emotional distress in caregivers was examined in one study; depression and hopelessness in caregivers were correlated with hopelessness in patients. ${ }^{53}$

The review also presented strong evidence for the following variables: social support and connections, quality of life, control or self-efficacy, as well as adjustment and resilience.

Social support or connections was examined in 10 studies. Seven of these studies indicated state and trait hope to be positively associated with social support, ${ }^{35} 41 \quad 46484952 \quad 62$ specifically support from family, ${ }^{41} 49{ }^{62}$ spouses $^{48}$ and friends. ${ }^{41}$ However, support from medical professionals was not a significant factor. ${ }^{41}$ Four studies examined social connections; ${ }^{35} 384262$ state and trait hope was positively correlated to relationships with neighbours and family, ${ }^{42}$ social relational quality ${ }^{35}$ and satisfaction with nursing care ${ }^{62}$ while hopelessness was associated with poor satisfaction with confidants. ${ }^{38}$

Significant correlations between hope or hopelessness and quality of life were reported in all five studies that examined this association; hope was associated with better quality of life ${ }^{4248}$ and spiritual well-being, ${ }^{63}$ while hopelessness was associated with poorer quality of life ${ }^{43}$ and spiritual well-being. ${ }^{47}$

All five studies that examined control or self-efficacy reported significant associations. ${ }^{54} 55586065$ Hope was significantly correlated with higher perception of control, ${ }^{55}$ higher internal locus of control, lower chance health locus of control ${ }^{58} \quad 60$ and generalised selfefficacy. ${ }^{54}$ Hopelessness was negatively correlated with self-efficacy in emotional regulation, managing physical symptoms and aspects of daily living. ${ }^{65}$

All four studies that examined adjustment and resilience reported significant associations; these indicated hope with psychosocial adjustment, ${ }^{44}$ resilience ${ }^{48}$ and post-traumatic growth, ${ }^{39}$ and hopelessness with poorer life adjustment ability. ${ }^{50}$

Uncertainty, demoralisation and resignation, as well as self-esteem, were examined in a small number of studies, with significant associations reported in all studies. In the three studies that examined uncertainty, hope was negatively associated with uncertainty, ${ }^{41} 4251$ such as in areas of symptoms, diagnosis, treatment and prognosis. ${ }^{51}$ Demoralisation and resignation were examined in three studies; ${ }^{45} 5764$ higher hopelessness was significantly associated with increased likelihood of demoralisation, ${ }^{57}$ resignation ${ }^{45}$ and desire for hastened death. ${ }^{64}$ In the two studies that examined self-esteem, hope was significantly associated with higher self-esteem. ${ }^{42} 49$

There was some evidence for coping responses and personality traits as correlates of hope and hopelessness. However, as different studies focused on different types of coping responses and traits, the results were inconclusive. Coping responses were examined in four studies. ${ }^{15} 424546$ While hope was significantly correlated to problem solving ${ }^{42}$ and coping that was optimistic, confrontative and self-reliant, ${ }^{46}$ it was negatively associated with catastrophic thinking ${ }^{15}$ as well as fatalistic and emotional coping. ${ }^{46}$ Hopelessness was negatively associated with a positive attitude towards cancer. ${ }^{45}$ Four studies examined personality traits: hope was significantly correlated to optimism, ${ }^{35} 3954$ while hopelessness was linked to higher neuroticism and lower extraversion. ${ }^{38}$

\section{DISCUSSION}

Overall, the literature on hope in Asian patients with cancer is still largely observational, and mostly restricted to the East Asia region (China, Hong Kong, Taiwan, Japan and Korea).

A majority of the studies in the current review used the HHI to measure hope or the 'helplessness/hopelessness' subscale of the MAC and Mini-MAC to measure hopelessness. The use of a variety of scales in the rest of the studies suggests that there is still room for standardising the measurement of hope and hopelessness to strengthen the evaluation of evidence across studies. ${ }^{12}$ The heterogeneity of measurements does, however, offer a greater understanding of hope as some scales measure trait hope, while others measure state hope. As there were only a few studies that include trait measurements, it is not yet possible to make any conclusive evaluations on the differences in association between trait and state hope (and hopelessness) in this review.

The current review extends knowledge from previous reviews by highlighting other associated sociodemographic variables, such as education level and employment. These variables were not highlighted in the previous reviews, ${ }^{412} 16$ but were consistently supported in the current review, and could thus be unique to the Asian cancer populations. The current review further supports the findings of previous reviews ${ }^{412} 16$ that hope is associated with socioeconomic status as well as positive clinical and psychosocial outcomes, while lower hope (and higher hopelessness) is associated with depression, anxiety, fatigue and demoralisation.

Furthermore, in the current review, cancer stage was significantly associated with hope and hopelessness in 8 of 11 studies. Out of these, five studies examined hopelessness. This was in contrast with the earlier reviews by $\mathrm{Chi}^{16}$ and Butt, ${ }^{12}$ which only included studies on hope, and suggested that hope was not significantly related to 
cancer stage. If studies measuring hopelessness were excluded, the evidence of the relationship between stage of cancer and hope would be diminished, thus emphasising the advantage of examining hope and hopelessness.

The current review of cancer populations in Asian countries showed that there was an association between hope (and hopelessness) and pain intensity and interference. ${ }^{37} 515965$ In contrast, the review by $\mathrm{Chi},{ }^{16}$ which was mostly on Western populations, revealed no significant associations with pain. Hope could be influenced by cultural and religious beliefs, such as beliefs in an afterlife, which might not be captured by existing measurement tools. ${ }^{59}$ Likewise, attitudes towards pain and pain expressions could also be affected by cultural beliefs. $^{72}$ Though inconclusive, these inconsistencies suggest that the relationship with cancer stage and pain may be nuanced by cultural differences; as such, it is necessary to validate the theoretical understanding of hope in a population before translating this into an intervention. As hope research is not as well developed in the Asian region, more work needs to be done to understand the conceptualisation and measurement of hope in the Asian setting.

The present review also presented inconsistent evidence regarding awareness of cancer diagnosis, with awareness of diagnosis associated with increased levels of hope in two studies, ${ }^{58}{ }^{60}$ but higher levels of hopelessness in one study. ${ }^{53}$ Qualitative studies examining awareness of diagnosis also produced mixed findings. Being told the truth was related to hope for some patients in one study as it alleviated their anxiety regarding their illness, ${ }^{73}$ but patients from another study highlighted that awareness of disease dampened hope and future outlook. ${ }^{74}$ The issue of disclosure of cancer diagnosis had been an ongoing debate in the Asian setting. ${ }^{75} 76$ Asian family members often did not want to disclose cancer diagnoses to patients to protect them from distress, ${ }^{53} 5875$ but patients themselves often expressed a desire to know the truth. ${ }^{77} 78$ With such conflicting results, it would thus be important to further examine whether awareness of diagnosis contributes to hope or hopelessness in Asian countries, in order to help physicians in discussing the cancer diagnoses and prognoses with patients.

\section{Limitations}

Some potentially relevant articles, which were mainly published in journals from Asian countries, were not included as the full text was unobtainable, despite repeated attempts at contacting libraries and authors. Furthermore, the nature of the search limited selected articles to those with at least English titles. These could potentially exclude studies that would provide greater insight to hope in the Asian context.

Furthermore, it is important to note the diversity within Asia region. While this review focused only on East, South and Southeast Asia countries for cultural homogeneity, this region already comprises various ethnic groups with diverse cultures and beliefs, which might influence the associations between hope and other factors. Furthermore, as a majority of the included studies were conducted in the East Asia region, this current review might be limited in generalising its findings to the entire Asia region.

Directionality of observed associations cannot be ascertained as the majority of studies were cross-sectional. Only four longitudinal studies were identified but temporal changes across the various parameters and hope or hopelessness had not been explored.

Another limitation of this study is the heterogeneity of the patient populations studied (in terms of age and cancer types), and heterogeneity in factors assessed and the instruments used to assess hope and hopelessness across the studies. Such heterogeneity prevented direct comparisons of the results across studies, limiting the conclusiveness of the review.

\section{Future directions}

The findings suggest several directions for future research. First, there is still considerably little research on correlates of hope and hopelessness in patients with cancer being conducted in South and Southeast Asia when compared to East Asia. Conducting more crosscultural studies could provide a better understanding of the variations in the relationship between hope and other related factors, allowing translation into more culturally sensitive psychosocial interventions to enhance coping with the cancer illness.

The inclusion of hopelessness in the current review demonstrated the advantages of examining hope and its antithetical concept-hopelessness, which could be recommended for future reviews in order to gain a comprehensive understanding of hope.

In addition, a greater focus on longitudinal studies would enable an assessment of changes in hope and hopelessness and their related factors over time and disease progression.

Overall the associations between hope and each of the factors highlighted above were supported by a majority of the studies that examined these factors. However, these associations exhibit small to moderate effect sizes, and conclusions within each individual study could be influenced by hidden confounders. Future studies should thus seek to examine the factors in a single study, or a meta-analysis could be conducted to examine the interplay of the different biopsychosocial factors in association with hope and hopelessness.

\section{Author affiliations}

${ }^{1}$ Department of Psychological Medicine, National University of Singapore, Singapore, Singapore

${ }^{2}$ Department of Psychological Medicine, National University Hospital, Singapore, Singapore

${ }^{3}$ Duke-NUS Medical School, Singapore, Singapore

${ }^{4}$ Department of Psychology, National University of Singapore, Singapore, Singapore 
Twitter Follow Haikel Lim at @haikelim

Acknowledgements The authors would like to thank Ms Serene Ang and Mr Gerrard Lai for their assistance rendered in this study.

Contributors RM conceptualised the project and reviewed the articles. SMC and IJY conducted the data abstraction, review of articles and analysis. SMC, HAL and JYST prepared the manuscript. EHK and KG were involved in the review of the manuscript. All authors read and approved the final manuscript.

Funding This research was supported by the National University of Singapore Start-Up Grant to RM (grant numbers R-177-000-039-133 and R-177-000039-733).

Disclaimer The study sponsor had no role in study design, data extraction and analyses, manuscript preparation or the decision to submit the manuscript for publication.

Competing interests None declared.

Provenance and peer review Not commissioned; externally peer reviewed.

Data sharing statement No additional data are available.

Open Access This is an Open Access article distributed in accordance with the Creative Commons Attribution Non Commercial (CC BY-NC 4.0) license, which permits others to distribute, remix, adapt, build upon this work noncommercially, and license their derivative works on different terms, provided the original work is properly cited and the use is non-commercial. See: http:// creativecommons.org/licenses/by-nc/4.0/

\section{REFERENCES}

1. Folkman S. Stress, coping, and hope. Psychological aspects of cancer: a guide to emotional and psychological consequences of cancer, their causes and their management. Springer, 2013.

2. Schrank B, Stanghellini G, Slade M. Hope in psychiatry: a review of the literature. Acta Psychiatr Scand 2008;118:421-33.

3. Herth K. Abbreviated instrument to measure hope: development and psychometric evaluation. J Adv Nurs 1992;17:1251-9.

4. Olver IN. Evolving definitions of hope in oncology. Curr Opin Support Palliat Care 2012;6:236-41.

5. Farran CJ, Herth KA, Popovich JM. Hope and hopelessness: critical clinical constructs. Sage Publications, Inc, 1995.

6. Clayton JM, Butow PN, Arnold RM, et al. Fostering coping and nurturing hope when discussing the future with terminally ill cancer patients and their caregivers. Cancer 2005;103:1965-75.

7. Gilbert MD. The resilient cancer patient. In: Duffy JD, Valentine AD, eds. MD Anderson manual of psychosocial oncology. New York (NY): McGraw-Hill Medical, 2011:35-46.

8. Petticrew M, Bell R, Hunter D. Influence of psychological coping on survival and recurrence in people with cancer: systematic review. BMJ 2002;325:1066.

9. Snyder CR. Hope theory: rainbows in the mind. Psychol Inq 2002;13:249-75.

10. Snyder CR. Conceptualizing, measuring, and nurturing hope. J Couns Dev 1995;73:355-60.

11. Gil S, Gilbar O. Hopelessness among cancer patients. J Psychosoc Oncol 2001;19:21-33.

12. Butt CM. Hope in adults with cancer: state of the science. Oncol Nurs Forum 2011;38:E341-50.

13. Beck AT, Weissman A, Lester D, et al. The measurement of pessimism: the hopelessness scale. J Consult Clin Psychol 1974;42:861

14. Snyder CR, Wrobleski KK, Parenteau SC, et al. Hope and hopelessness. In: Haas LJ, ed. Handbook of primary care psychology. New York: Oxford University Press, 2004:145-55.

15. Lai YH, Chang JT, Keefe FJ, et al. Symptom distress, catastrophic thinking, and hope in nasopharyngeal carcinoma patients. Cancer Nurs 2003;26:485-93.

16. Chi GC. The role of hope in patients with cancer. Oncol Nurs Forum 2007:34:415-24.

17. Moher D, Cook DJ, Eastwood S, et al. Improving the quality of reports of meta-analyses of randomised controlled trials: the QUOROM statement. Lancet 1999;354:1896-900.

18. Moher D, Liberati A, Tetzlaff J, et al. Preferred reporting items for systematic reviews and meta-analyses: the PRISMA statement. Ann Intern Med 2009;151:264-9.
19. Lord K, Ibrahim K, Kumar S, et al. Are depressive symptoms more common among British South Asian patients compared with British White patients with cancer? A cross-sectional survey. BMJ Open 2013;3:e002650.

20. Roy R, Symonds RP, Kumar DM, et al. The use of denial in an ethnically diverse British cancer population: a cross-sectional study. Br J Cancer 2005;92:1393-7.

21. Kylmä J, Vehviläinen-Julkunen K. Hope in nursing research: a meta-analysis of the ontological and epistemological foundations of research on hope. J Adv Nurs 1997;25:364-71.

22. Hong IWM, Ow R. Hope among terminally ill patients in Singapore: an exploratory study. Soc Work Health Care 2007;45:85-106.

23. Vaughn LM, Jacquez F, Baker RC. Cultural health attributions, beliefs, and practices: effects on healthcare and medical education. Open Med Educ J 2009;2:64-74.

24. Sankaranarayanan R, Ramadas K, Qiao YL. Managing the changing burden of cancer in Asia. BMC Med 2014;12:3.

25. Grassi L, Caruso R, Sabato S, et al. Psychosocial screening and assessment in oncology and palliative care settings. Front Psychol 2014;5:1485.

26. Mak MHJ. Accepting the timing of one's death: an experience of Chinese hospice patients. Omega 2002;45:245-60

27. Kissane DW, Lethborg CE, Kelly B. Spiritual and religious coping with cancer. In: Grassi L, Riba M, eds. Clinical psycho-oncology: an international perspective, 2012:281-95.

28. Lim H, Yee I, Griva K, et al. The biopsychosocial correlates of hope in Asian cancer patients: a systematic review. PROSPERO, 2015: CRD42015014522.

29. Ito T. Clinical research of physician support and psychological responses after surgery for patients with non-small-cell lung cancer. Tokyo Jikeikai Med J 2003;118:321-32.

30. Kubo S. On nursing education. 14. Hope for life in patients with terminal cancer. Kango Kyoiku 1983;24:500

31. Ruijie Z, Changde J, Li J. Research status quo of hope level of cancer patients [J]. Chin Nurs Res 2013;4:006.

32. Wen YL, Wen $\mathrm{CH}$, Wang KY, et al. Resilience in cancer patients and related nursing interventions. Hu Li Za Zhi 2013;60:93.

33. Lie DA, Lee-Rey E, Gomez A, et al. Does cultural competency training of health professionals improve patient outcomes? A systematic review and proposed algorithm for future research. $J$ Gen Intern Med 2011;26:317-25.

34. Sallis JF, Prochaska JJ, Taylor WC. A review of correlates of physical activity of children and adolescents. Med Sci Sports Exerc 2000;32:963-75.

35. Hou WK, Law CC, Yin J, et al. Resource loss, resource gain, and psychological resilience and dysfunction following cancer diagnosis: a growth mixture modeling approach. Health Psychol 2010;29:484-95.

36. Nagano J, Ichinose $\mathrm{Y}$, Asoh $\mathrm{H}$, et al. A prospective Japanese study of the association between personality and the progression of lung cancer. Intern Med 2006;45:57-63.

37. Nakaya N, Saito-Nakaya K, Akechi T, et al. Negative psychological aspects and survival in lung cancer patients. Psychooncology 2008;17:466-73.

38. Uchitomi Y, Akechi T, Fujimori M, et al. Mental adjustment after surgery for non-small cell lung cancer. Palliat Support Care 2003;1:61-70

39. Ho S, Rajandram RK, Chan N, et al. The roles of hope and optimism on posttraumatic growth in oral cavity cancer patients. Oral Oncol 2011;47:121-4.

40. Shimizu K, Nakaya N, Saito-Nakaya K, et al. Clinical biopsychosocial risk factors for depression in lung cancer patients: a comprehensive analysis using data from the lung cancer database project. Ann Oncol 2012;23:1973-9.

41. Hwang $\mathrm{R}, \mathrm{Ku} \mathrm{N}$, Mao $\mathrm{H}$, et al. Hope and related factors of breast cancer women [Chinese]. Nurs Res 1996;4:35-46.

42. Jo KH, Son BK. [The relationship of uncertainty, hope and quality of life in patients with breast cancer]. Taehan Kanho Hakhoe chi 2004;34:1184-93.

43. Kim SW, Kim SY, Kim JM, et al. Relationship between a hopeful attitude and cellular immunity in patients with breast cancer. Gen Hosp Psychiatry 2011;33:371-6.

44. Lee EH. Fatigue and hope: relationships to psychosocial adjustment in Korean women with breast cancer. Appl Nurs Res 2001;14:87-93.

45. Ueta I, Onishi C. Coping strategies and psychological adjustment among breast cancer patients with recurrence/metastasis [Japanese]. J Jpn Acad Nurs Sci 2011;31:42-51.

46. Zhang J, Gao W, Wang P, et al. Relationships among hope, coping style and social support for breast cancer patients. Chin Med J 2010;123:2331-5. 
47. Raja Lexshimi RG, Mohd Fahmi E, Lee SC, et al. Spirituality and mental adjustment as coping strategies among women with breast cancer. Malays J Public Health Med 2014;14:1-9.

48. Ryu YM, Yi M. The factors influencing quality of life in women with breast cancer [Korean]. Asian Oncol Nurs 2013;13:121-7.

49. Tae YS, Heitkemper M, Kim MY. A path analysis: a model of depression in Korean women with breast cancer-mediating effects of self-esteem and hope. Oncol Nurs Forum 2012;39:E49-57.

50. Horii N, Maekawa A. Development of a nursing model for life adjustment in patients with lung cancer in Japan. Nurs Health Sci 2013;15:300-8.

51. Hsu TH, Lu MS, Tsou TS, et al. The relationship of pain, uncertainty, and hope in Taiwanese lung cancer patients. $J$ Pain Symptom Manage 2003;26:835-42.

52. Chen $\mathrm{H}$, Wang $\mathrm{H}$. The relationship between hope, social support and demography of hospitalized patients with hemolymph neoplasm [Chinese]. Nurs Res 1997;5:487-98.

53. Han Y, Yuan J, Luo Z, et al. Determinants of hopelessness and depression among Chinese hospitalized esophageal cancer patients and their family caregivers. Psychooncology 2013;22:2529-36.

54. Yang YL, Liu L, Wang XX, et al. Prevalence and associated positive psychological variables of depression and anxiety among Chinese cervical cancer patients: a cross-sectional study. PLoS ONE 2014;9:e94804.

55. Chang LC, Li IC. The correlation between perceptions of control and hope status in home-based cancer patients. J Res Nurs 2002;10:73-82.

56. Chen ML. Pain and hope in patients with cancer: a role for cognition. Cancer Nurs 2003;26:61-7.

57. Lee CY, Fang CK, Yang YC, et al. Demoralization syndrome among cancer outpatients in Taiwan. Support Care Cancer 2012;20:2259-67.

58. Lin CC, Tsai HF, Chiou JF, et al. Changes in levels of hope after diagnostic disclosure among Taiwanese patients with cancer. Cancer Nurs 2003:26:155-60.

59. Lin CC, Lai YL, Ward SE. Effect of cancer pain on performance status, mood states, and level of hope among Taiwanese cancer patients. J Pain Symptom Manage 2003;25:29-37.

60. Lin CC, Tsay HF. Relationships among perceived diagnostic disclosure, health locus of control, and levels of hope in Taiwanese cancer patients. Psychooncology 2005;14:376-85.

61. Shun SC, Hsiao FH, Lai YH. Relationship between hope and fatigue characteristics in newly diagnosed outpatients with cancer. Oncol Nurs Forum 2011;38:E81-6.

62. Chae SY, Kim KH. Physical symptoms, hope and family support of cancer patients in the general hospitals and long-term care hospitals. Korean J Adult Nurs 2013;25:298-311.
63. Jun SY, Ko IS. Relationship of spiritual well-being, hope on fatigue in cancer patients on chemotherapy. Korean J Adult Nurs 2012;24:557-68.

64. Shim EJ, Hahm BJ. Anxiety, helplessness/hopelessness and 'desire for hastened death' in Korean cancer patients. Eur J Cancer Care 2011;20:395-402.

65. Ueda S, Katsuno T. Factors influencing the psychological adjustment to cancer among elderly patients-perception of physical symptoms, physical condition, and self-efficacy [Japanese]. J Jpn Acad Nurs Sci 2009;29:52-9.

66. Nowotny M. Assessment of hope in patients with cancer: development of an instrument. Oncol Nurs Forum 1988;16:57-61.

67. Kim DS, Lee SW. Development of an instrument to measure hope for the cancer patients. J Korean Acad Nurs 1998;28:441-56.

68. Snyder CR, Harris C, Anderson JR, et al. The will and the ways: development and validation of an individual-differences measure of hope. J Pers Soc Psychol 1991;60:570.

69. Watson M, Greer S, Young J, et al. Development of a questionnaire measure of adjustment to cancer: the MAC scale. Psychol Med 1988;18:203-9.

70. Watson M, Law MG, Santos MD, et al. The Mini-MAC: further development of the mental adjustment to cancer scale. J Psychosoc Oncol 1994;12:33-46.

71. Grossarth-Maticek R, Eysenck HJ. Personality, stress and disease: description and validation of a new inventory. Psychol Rep 1990;66:355-73.

72. Chen $\mathrm{CH}$, Tang ST, Chen $\mathrm{CH}$. Meta-analysis of cultural differences in Western and Asian patient-perceived barriers to managing cancer pain. Palliat Med 2012;26:206-21.

73. Hsiao SM, Gau ML, Ingleton C, et al. An exploration of spiritual needs of Taiwanese patients with advanced cancer during the therapeutic processes. J Clin Nurs 2011;20:950-9.

74. Aihara $\mathrm{Y}$, Sato $\mathrm{E}$, Hashimoto $\mathrm{H}$, et al. Hopes of people in their twenties and thirties living with leukemia/lymphoma [Japanese] $J$ Jpn Acad Nurs Sci 2004;24:83-91.

75. Back MF, Huak CY. Family centred decision making and non-disclosure of diagnosis in a South East Asian oncology practice. Psychooncology 2005;14:1052-9.

76. Tanida N. Japanese attitudes towards truth disclosure in cancer. Scand J Public Health 1994;22:50-7.

77. Tang ST, Liu TW, Lai MS, et al. Congruence of knowledge, experiences, and preferences for disclosure of diagnosis and prognosis between terminally-ill cancer patients and their family caregivers in Taiwan. Cancer Invest 2006;24:360-6.

78. Yun YH, Lee CG, Kim SY, et al. The attitudes of cancer patients and their families toward the disclosure of terminal illness. J Clin Oncol 2004;22:307-14. 